\title{
Within-person pain variability and physical activity in older adults with osteoarthritis from six European countries
}

Erik J. Timmermans ${ }^{1 *}$ D, Elisa J. de Koning ${ }^{1}$, Natasja M. van Schoor ${ }^{1}$, Suzan van der Pas ${ }^{1}$, Michael D. Denkinger ${ }^{2}$, Elaine M. Dennison ${ }^{3}$, Stefania Maggi ${ }^{4}$, Nancy L. Pedersen ${ }^{5}$, Ángel Otero ${ }^{6}$, Richard Peter ${ }^{7}$, Cyrus Cooper $^{3}$, Paola Siviero ${ }^{4}$, Maria Victoria Castell ${ }^{6}$, Florian Herbolsheimer ${ }^{7}$, Mark Edwards $^{3}$, Federica Limongi ${ }^{4}$,

Dorly J. H. Deeg ${ }^{1}$ and Laura A. Schaap ${ }^{8}$

\begin{abstract}
Background: This study examines the association of both pain severity and within-person pain variability with physical activity $(P A)$ in older adults with osteoarthritis $(O A)$.

Methods: Data from the European Project on OSteoArthritis were used. At baseline, clinical classification criteria of the American College of Rheumatology were used to diagnose OA in older adults (65-85 years). At baseline and 12-18 months follow-up, frequency and duration of participation in the activities walking, cycling, gardening, light and heavy household tasks, and sports activities were assessed with the Longitudinal Aging Study Amsterdam Physical Activity Questionnaire. Physical activity was calculated in kcal/day, based on frequency, duration, body weight and the metabolic equivalent of each activity performed. At baseline and 12-18 months follow-up, pain severity was assessed using the pain subscales of the Western Ontario and McMaster Universities OA Index and the Australian/Canadian Hand OA Index. Within-person pain variability was assessed using two-week pain calendars that were completed at baseline, 6 months follow-up and 12-18 months follow-up.
\end{abstract}

Results: Of all 669 participants, 70.0\% were women. Sex-stratified multiple linear regression analyses showed that greater pain severity at baseline was cross-sectionally associated with less PA in women (Ratio $=0.95,95 \% \mathrm{Cl}=0.90-0$. 99), but not in men (Ratio $=0.99,95 \% \mathrm{Cl}=0.85-1.15$ ). The longitudinal analyses showed a statistically significant inverse association between pain severity at baseline and PA at follow-up in women (Ratio $=0.94,95 \% \mathrm{Cl}=0.89-0.99$ ), but not in men (Ratio $=1.00,95 \% \mathrm{Cl}=0.87-1.11$ ). Greater pain variability over $12-18$ months was associated with more PA at follow-up in men (Ratio $=1.18,95 \% \mathrm{Cl}=1.01-1.38$ ), but not in women (Ratio $=0.94,95 \% \mathrm{Cl}=0.86-1.03$ ).

Conclusions: Greater pain severity and less pain variability are associated with less PA in older adults with OA. These associations are different for men and women. The observed sex differences in the various associations should be studied in more detail and need replication in future research.

Keywords: European multi-cohort study, Older adults, Osteoarthritis, Pain variability, Pain severity, Physical activity

\footnotetext{
* Correspondence: ej.timmermans@vumc.nl

${ }^{1}$ Department of Epidemiology and Biostatistics, Amsterdam University

Medical Center, VU University Medical Center, Amsterdam Public Health

Research Institute, De Boelelaan 1089A, 1081HV, Amsterdam, Netherlands

Full list of author information is available at the end of the article
}

(c) The Author(s). 2019 Open Access This article is distributed under the terms of the Creative Commons Attribution 4.0 International License (http://creativecommons.org/licenses/by/4.0/), which permits unrestricted use, distribution, and reproduction in any medium, provided you give appropriate credit to the original author(s) and the source, provide a link to the Creative Commons license, and indicate if changes were made. The Creative Commons Public Domain Dedication waiver (http://creativecommons.org/publicdomain/zero/1.0/) applies to the data made available in this article, unless otherwise stated. 


\section{Background}

Osteoarthritis (OA) is one of the most common musculoskeletal disorders worldwide [1]. The condition is a leading cause of disability and a major contributor to loss of independence among older adults, with joint pain representing one of the key debilitating symptoms [1-3]. While OA is characterized as a slowly progressive disease, acute flares, episodes of severe pain, and substantial fluctuations in pain intensity are part of the natural course of the condition $[4,5]$. It has been suggested that physical activity (PA) reduces joint pain in older adults with $\mathrm{OA}$, and helps them to maintain or improve functioning [6-10]. Despite the potential health benefits of PA are well established, the majority of older people with OA are not sufficiently physically active [11-16]. Besides the fear of continuing or worsening wear and tear within the joint, it has been suggested that the most important reason for older adults with OA to avoid physical activities is pain that is associated with initiating activities [15, 17-19].

Pain patterns differ substantially across individuals with OA $[4,5,20,21]$. Several studies have shown that higher levels of pain severity are associated with less PA in older people with OA $[15,18,19,22,23]$. Although joint pain often fluctuates in older adults with OA, previous studies mainly focused on the impact of momentary joint pain severity on PA and did not investigate the association between joint pain variability and PA in these individuals.

The fluctuating course of joint pain in OA requires repeated measurements and is difficult to capture in research based on commonly used extensive instruments, such as the Western Ontario and McMaster Universities OA Index (WOMAC) and the Australian/Canadian Hand Osteoarthritis Index (AUSCAN) [24-26]. Pain variability can more easily be measured using pain calendars (i.e., pain diaries) over a large period of time. In the European Project on OSteoArthritis (EPOSA), WOMAC, AUSCAN as well as pain calendars have been used to assess pain [27]. The EPOSA study provides a unique opportunity to assess the association of both pain severity and within-person pain variability with PA in older adults with $\mathrm{OA}$ in the general population across six European countries, including both older adults with mild and severe OA, and those seeking care and not seeking care [27]. Assuming that a lack of predictability of pain is detrimental to PA [18], it is hypothesized that greater pain severity and greater pain variability over time is associated with less PA in these individuals.

\section{Methods}

\section{Design and study sample}

In the present study, data from the European Project on OSteoArthritis (EPOSA) were used. The main objective of the EPOSA study is to investigate the personal and societal burden of $\mathrm{OA}$ and its determinants in older adults in six European countries. The study design and data collection are described in detail elsewhere [27]. In summary, random samples were taken from existing population-based cohorts in five European countries (Germany, the Netherlands, Spain, Sweden, and the United Kingdom). In Italy, a new sample was drawn. In the EPOSA study, the data collection took place twice with 12-18 months between the baseline and the follow-up measurement. At baseline and 12-18 months follow-up, a total of 2942 (average response rate: $72.8 \%$ (range: 64.6-82.2\%)) and 2455 (average response rate: 83.4\% (range: 68.2-92.3\%)) older adults, respectively, participated in the EPOSA project. All participants were interviewed by a trained interviewer at home or in a clinical center, using a standardized questionnaire and a clinical exam. After the baseline and 12-18 months follow-up interview as well as 6 months after the baseline interview, participants were asked to complete a two-week calendar assessing joint pain. At baseline, the classification criteria of the American College of Rheumatology were used to diagnose clinical knee, hand and hip OA [28]. In total, 889 participants had knee, hand and/or hip OA at baseline. Of all participants with OA at baseline, 713 individuals also participated at follow-up. The final analytical sample consisted of 669 older adults with clinical OA who participated at baseline as well as at follow-up and did not report joint replacements at the site of the clinical OA. The study design and all procedures were approved by the Ethical Review Boards of the participating institutions. All participants provided written informed consent prior to the start of the study.

\section{Measurements \\ Dependent variable}

Physical activity At baseline and 12-18 months follow-up, physical activity (PA) was assessed using the Longitudinal Aging Study Amsterdam Physical Activity Questionnaire (LAPAQ) [29]. The LAPAQ examines the frequency and duration of participation in different activities (i.e., walking outside, cycling, gardening, light and heavy household tasks, and a maximum of two sports) during the previous two weeks. Physical activity was calculated in $\mathrm{kcal} /$ day, based on frequency, duration, body weight and the metabolic equivalent of each performed activity.

\section{Independent variables}

Pain severity At baseline and 12-18 months follow-up, pain severity in the past $48 \mathrm{~h}$ was measured with the 
WOMAC (knee and hip OA) and the AUSCAN (hand OA) pain subscales. The WOMAC and AUSCAN pain subscales are described in detail elsewhere [24, 25]. Because these pain subscales are scored on the same scale (score range: 0-20), the scores of knee, hip and hand pain were added up, resulting in a total joint pain severity score (score range: 0-60). For comparability to other studies [24-26], this WOMAC/AUSCAN combined pain score was transformed to a $0-100$ scale. A higher WOMAC/AUSCAN combined pain score indicates more joint pain.

Pain variability Pain variability was assessed with two -week pain calendars completed by participants immediately after the baseline interview, six months later and after the 12-18 months follow-up interview. Participants from Spain did not complete the pain calendar at 6-months follow-up. Per day, participants indicated how much joint pain they experienced on an 11-point rating scale (score range: $0-10)$. On this rating scale, a score of 0 represents no pain and a score of 10 indicates extreme pain. For each participant, the standard deviation (SD) of the scores from all available days of the three calendars was used as measure of individual day-to-day pain variability. A larger individual SD score indicates more day-to-day pain variability within a person. At least 7 of 14 days of at least one calendar had to be completed by a participant in order to be included in the pain variability analyses. This approach corresponds with previous research [26].

Potential effect modifiers and confounders Age, sex $(0=$ men, $1=$ women $)$, and country of residence were considered as potential effect modifiers in the association between pain and PA [30-32]. Age was categorized into younger-old people and older-old people on the basis of $1-2$ quartiles (65.0-74.0 years) and 3-4 quartiles (74.1-85.0 years), respectively. Country of residence was dummy-coded and included: Germany, Italy, the Netherlands, Spain, Sweden, and the United Kingdom. Sweden was considered as the reference category, because the Swedish respondents reported, on average, to be physically less active.

The following potential confounders were considered in this study: age in years, sex, country of residence (if no effect modifiers), educational level, smoking, alcohol consumption, weight status, number of chronic diseases, and use of pain medications.

Educational level was classified into four categories: no education (reference category; elementary school not completed), low (elementary school completed), intermediate (vocational or general secondary education), and high (college or university education).
Smoking status was self-reported and classified into three categories: never smokers (reference category), former smokers, and current smokers. Alcohol consumption was self-reported $(0=$ no, $1=$ yes $)$.

Weight status was assessed using the Body Mass Index, which was calculated as weight in kilograms divided by height in meters squared [33]. The weight of participants was measured to the nearest $0.1 \mathrm{~kg}$ using a calibrated scale. The height of participants was measured to the nearest $0.001 \mathrm{~m}$ using a stadiometer.

Number of chronic diseases was assessed by asking for the presence of the following chronic diseases: chronic non-specific lung disease, cardiovascular diseases, peripheral artery diseases, diabetes mellitus, stroke, cancer, and osteoporosis.

Medication use $(0=$ no, $1=$ yes $)$ referred to the use of analgesics (Anatomical Therapeutic Chemical classification: N02 subgroup) and/or anti-inflammatory and anti-rheumatic products (Anatomical Therapeutic Chemical classification: M01 subgroup).

Statistical analyses Characteristics of the study sample were presented using descriptive statistics. Means and SDs were presented for normally distributed continuous variables. Medians and interquartile ranges (IQRs) were presented for skewed continuous variables. Frequencies and proportions were presented for dichotomous or categorical variables. With the exception of age, sex and country, all descriptive statistics were weighted to adjust for differences in the distributions of age and sex across the samples of the six cohort studies. Weights were calculated per sex and per five-year age category, using the formula $W=N_{\exp } / N_{\text {obs }}$, where $\mathrm{N}_{\exp }$ is the number of persons in a specific age/sex category in the European population and $\mathrm{N}_{\mathrm{obs}}$ is the number of persons in a specific age/sex category in the cohort [26, 34].

Multiple linear regression analyses were used to examine the associations between each pain measure and PA. To study possible effect modification by age, sex, and country of residence, an interaction term of the determinant with the potential effect modifier was created. This interaction term was added to the model. If the $p$-value of the interaction term was below 0.10 , effect modification was considered to be present and stratified analyses were conducted [35]. In case it turned out that age, sex or country of residence was no effect modifier, the variable was considered as confounder in the subsequent analyses.

The associations between the pain measures and PA were constructed step by step in two models. In Model 1, we adjusted for age, sex, and country of residence (if no effect modifiers). In Model 2, we additionally adjusted for educational level, smoking, alcohol consumption, weight 
status, number of chronic diseases, and use of medications.

For pain severity, both cross-sectional and longitudinal multiple linear regression analyses were conducted. For the cross-sectional analysis, the baseline WOMAC/AUSCAN combined pain score was the main determinant and PA at baseline the continuous outcome measure. To examine the prospective association between pain severity and PA, the baseline WOMAC/AUSCAN combined pain score was the main determinant and PA at follow-up the outcome measure. In this analysis, PA at baseline was used as additional covariate.

To study whether pain fluctuations over time affect PA, multiple linear regression analyses were conducted with the individual joint pain SDs (calculated from the three two-week pain calendars at baseline, 6 months follow-up and 12-18 months follow-up) as the main determinant and PA at 12-18 months follow-up as outcome measure.

Three pre-planned sensitivity analyses were conducted. A potential disadvantage of using the SD of the individual pain intensity scores from all available pain calendar days as measure of day-to-day pain variability is that the $\mathrm{SD}$ is the same for individuals who report the same extent of fluctuations, regardless of their mean pain intensity score [26]. For example, two persons with mean pain intensity levels of 0 and 10, respectively, who experience no pain fluctuations, will both have a SD of 0 . In the first pre-planned sensitivity analysis, we repeated the pain variability analysis whilst leaving out persons with no or only little reported joint pain (mean pain score on at least one calendar $\leq 2$ ).

Hip and knee OA might be more strongly related to the physical activities that are assessed by the LAPAQ. In the second pre-planned sensitivity analysis, we repeated all analyses whilst leaving out persons with only hand OA. It is expected that these analyses reveal stronger associations of both pain severity and within-person pain variability with PA than those in which also participants with hand OA are included.

In the present study, the pain calendar at 12-18 months follow-up was completed in the two weeks after the PA assessment. However, we defined PA as our dependent variable. In the third pre-planned sensitivity analysis, we repeated the pain variability analyses whilst leaving out the 12-18 months pain calendar.

Because the distribution of PA was skewed to the right, we performed a natural log transformation on the LAPAQ data (i.e., $\operatorname{Ln}(1+\mathrm{LAPAQ}$ score) in the present study. The regression coefficients and confidence intervals (CIs) from the multiple linear regression analyses were back-transformed to obtain interpretable ratios. From these ratios, a percentage of change in the outcome variable (PA) per one-unit change in the determinant (pain) can be derived.
Because a one-unit change on the pain severity scale of 0 100 is very small, we derived a more meaningful 10-unit change in the present study [36]. A two-sided $p$-value below 0.05 was considered as statistically significant. All analyses were conducted with IBM SPSS Statistics for Windows, Version 22.0 [37].

\section{Results}

The characteristics of the study sample are presented in Table 1 . The mean age of all 669 participants at baseline was $73.9(\mathrm{SD}=4.9)$ years with an age-range of $65-85$ years. The majority $(70.0 \%)$ of participants was woman. Of all 669 participants, $62.9 \%$ had knee OA, 19.9\% had hip OA, and 56.5\% had hand OA. At baseline and $12-$ 18 months follow-up, the average pain severity was 18.1 $(\mathrm{SD}=12.3)$ and $17.9(\mathrm{SD}=15.0)$ on the WOMAC/AUSCAN combined pain score, respectively. The participants completed, on average, $34.2(\mathrm{SD}=9.2) \quad$ (range $=7-42$ days) days of the pain calendars. In the full sample, the average pain variability on the pain calendars was 1.4 $(\mathrm{SD}=0.8)$ individual standard deviation units. At baseline and 12-18 months follow-up, the participants spent $691.5(\mathrm{IQR}=437.4-1043.4)$ and $664.0 \quad(\mathrm{IQR}=417.5-$ 1077.3) $\mathrm{kcal} /$ day on PA, respectively (Table 1 ).

In all analyses, sex was found to be a significant effect modifier and, therefore, all analyses were stratified for sex. Age and country of residence were not found to be a significant effect modifier in all analyses.

\section{Cross-sectional analyses: pain severity and physical activity}

After adjustment for age and country of residence, the cross-sectional baseline association between pain severity and PA was statistically significant in women (Ratio $=0.94$, 95\% CI $=0.89-0.98$ ) (Table 2; Model 1). After additional adjustment for all other confounders, the association between pain severity and PA remained statistically significant in women (Ratio $=0.95,95 \% \mathrm{CI}=0.90$ 0.99). Accordingly, a 10-unit higher pain severity score is associated with 5\% less PA (Table 2; Model 2). No statistically significant association was observed between pain severity and PA in men (Table 2).

\section{Longitudinal analyses: pain severity and physical activity} After adjustment for age, country of residence and PA at baseline, the prospective association between pain severity at baseline and PA at 12-18 months follow-up was statistically significant in women (Ratio $=0.94,95 \% \mathrm{CI}=$ 0.89-0.99) (Table 3; Model 1). After additional adjustment for all other confounders, this association remained unchanged in women (Ratio $=0.94,95 \% \mathrm{CI}=$ 0.89-0.99) (Table 3; Model 1). Accordingly, a 10-unit higher pain severity score at baseline is associated with $6 \%$ less PA at follow-up in women. The prospective 
Table 1 Characteristics of the study sample $e^{a, b}$

\begin{tabular}{|c|c|c|c|}
\hline & Study sample $(n=669)$ & $\operatorname{Men}(n=211)$ & Women $(n=458)$ \\
\hline Age (in years) (Mean (SD)) & $73.9(4.9)$ & $74.5(5.1)$ & $73.7(4.8)$ \\
\hline \multicolumn{4}{|l|}{ Country (\%) } \\
\hline Germany & 8.4 & 9.5 & 7.9 \\
\hline Italy & 17.6 & 19.9 & 16.6 \\
\hline Netherlands & 16.6 & 14.7 & 17.4 \\
\hline Spain & 21.8 & 20.4 & 22.5 \\
\hline Sweden & 20.6 & 16.1 & 22.7 \\
\hline United Kingdom & 14.9 & 19.4 & 12.9 \\
\hline \multicolumn{4}{|l|}{ Educational level (\%) } \\
\hline No education & 14.4 & 12.0 & 15.3 \\
\hline Low & 35.4 & 35.1 & 35.5 \\
\hline Intermediate & 32.3 & 35.7 & 31.0 \\
\hline High & 17.9 & 17.2 & 18.2 \\
\hline \multicolumn{4}{|l|}{ Smoking (\%) } \\
\hline Never & 52.4 & 29.6 & 61.2 \\
\hline Former & 41.9 & 63.9 & 33.5 \\
\hline Current & 5.7 & 6.5 & 5.3 \\
\hline Alcohol consumption (yes) (\%) & 70.5 & 86.1 & 64.5 \\
\hline Weight status (BMl in $\mathrm{kg} / \mathrm{m}^{2}$ ) (Mean (SD)) & $28.5(5.0)$ & $28.4(3.9)$ & $28.5(5.4)$ \\
\hline Number of chronic diseases (Median (IQR)) & $1(0-2)$ & $1(0-2)$ & $1(0-2)$ \\
\hline $\begin{array}{l}\text { Use of analgesic and/or anti-inflammatory and } \\
\text { anti-rheumatic products (yes) (\%) }\end{array}$ & 32.7 & 33.0 & 32.6 \\
\hline \multicolumn{4}{|l|}{ Presence of clinical osteoarthritis (\%) } \\
\hline Knee osteoarthritis & 62.9 & 67.8 & 61.0 \\
\hline Hand osteoarthritis & 56.5 & 42.7 & 61.9 \\
\hline Hip osteoarthritis & 19.9 & 19.3 & 20.2 \\
\hline \multicolumn{4}{|l|}{ Pain severity at baseline (Mean (SD)) ${ }^{c}$} \\
\hline Baseline & $18.1(12.3)$ & $14.6(9.8)$ & $19.4(12.9)$ \\
\hline Follow-up & $17.9(15.0)$ & $13.7(13.4)$ & $19.6(14.4)$ \\
\hline Pain variability (Mean (SD)) ${ }^{d}$ & $1.4(0.8)$ & $1.4(0.9)$ & $1.4(0.8)$ \\
\hline \multicolumn{4}{|l|}{ Physical activity (in kcal/day) } \\
\hline \multicolumn{4}{|l|}{ (Median (IQR)) } \\
\hline Baseline & $691.5(437.4-1043.4)$ & $636.6(383.1-1090.1)$ & $704.0(447.7-1030.4)$ \\
\hline Follow-up & $664.0(417.5-1077.3)$ & $662.4(348.0-1146.2)$ & $667.1(436.8-1059.1)$ \\
\hline
\end{tabular}

a Abbreviations: BMI Body Mass Index, IQR Interquartile range, $n$ Number of participants, $k c a l$ Kilocalories, $\mathrm{kg} / \mathrm{m}^{2} \mathrm{kilogram}$ per square meter, OA Osteoarthritis, $S D$ Standard deviation

With exception of age and country, descriptive statistics are weighted

${ }^{\mathrm{C}} \mathrm{A}$ higher pain severity score indicates more pain

${ }^{\mathrm{d}}$ Pain variability is presented in individual standard deviation units. A higher pain variability score indicates more day-to-day pain fluctuations

association between pain severity at baseline and PA at 12-18 months follow-up was not statistically significant in men (Table 3).

\section{Longitudinal analyses: pain variability and physical activity}

A greater pain variability over $12-18$ months was significantly associated with more PA in men (Ratio $=1.18$, $95 \% \mathrm{CI}=1.01-1.38)$, but with less $\mathrm{PA}$ in women although not significant (Ratio $=0.94,95 \% \mathrm{CI}=0.86$ 1.03) (Table 4; Model 2). Accordingly, a 1-unit higher pain variability score is associated with $18 \%$ more PA in men, and with $6 \%$ less PA in women.

\section{Sensitivity analysis}

The various sensitivity analyses did not change any of the conclusions (results not shown). 
Table 2 Cross-sectional associations between pain severity and physical activity, stratified by sex ${ }^{a-d}$

\begin{tabular}{llllll}
\hline & Men & & & Women \\
\cline { 2 - 3 } & Ratio $(95 \% \mathrm{Cl})$ & $p$-value & & Ratio $(95 \% \mathrm{Cl})$ & $p$-value \\
\hline Model 1 & $1.00(0.86-1.16)$ & 0.97 & & $0.94(0.89-0.98)$ & 0.02 \\
Model 2 & $0.99(0.85-1.15)$ & 0.87 & & $0.95(0.90-0.99)$ & 0.04
\end{tabular}

${ }^{a}$ Abbreviation: $\mathrm{Cl}$ Confidence Interval

${ }^{b}$ In these analyses, the baseline WOMAC/AUSCAN combined pain score was the main determinant and physical activity at baseline the outcome measure 'Model 1: adjusted for age and country of residence

Model 2: additionally adjusted for educational level, smoking, alcohol consumption, weight status, number of chronic diseases, and use of analgesics and/or anti-inflammatory and anti-rheumatic products

${ }^{\mathrm{d}}$ The cross-sectional associations are displayed per 10 units increase in pain severity

\section{Discussion}

This study examined whether pain severity and pain fluctuations over time were associated with PA in older adults with OA from the general European population. The results showed that greater pain severity was associated with less PA in women, but not in men. Furthermore, the findings showed that greater pain variability was associated with more PA in men. Pain variability was not associated with PA in women. The various sensitivity analyses did not result in substantially different findings.

The findings of this study showed that greater pain severity was associated with less PA in older women. The observed difference in this association between men and women could be possibly explained by differences in coping strategies that are used by men and women [38]. Pain catastrophizing is a pain coping strategy referring to the exacerbation of pain-related information $[38,39]$, while self-efficacy refers to the belief of a person in his or her ability to achieve goals $[38,40]$. Previous studies have shown that catastrophizing is associated with pain and pain-related disability and that women use this coping strategy more often than men [38, 41]. Previous studies have also shown that men more often demonstrate higher levels of self-efficacy than women [38, 42].

Table 3 Longitudinal associations between pain severity and physical activity, stratified by sex ${ }^{a-d}$

\begin{tabular}{|c|c|c|c|c|}
\hline & \multicolumn{2}{|l|}{ Men } & \multicolumn{2}{|l|}{ Women } \\
\hline & Ratio (95\% Cl) & $\overline{p \text {-value }}$ & Ratio (95\% Cl) & $\overline{p \text {-value }}$ \\
\hline Model 1 & $0.99(0.88-1.11)$ & 0.86 & $0.94(0.89-0.99)$ & 0.03 \\
\hline Model 2 & $1.00(0.87-1.11)$ & 0.81 & $0.94(0.89-0.99)$ & 0.02 \\
\hline
\end{tabular}

abbreviation: $\mathrm{Cl}$ Confidence Interval

${ }^{\mathrm{b}}$ In these analyses, the baseline WOMAC/AUSCAN combined pain score was the main determinant and physical activity at 12-18 months follow-up the outcome measure

${ }^{c}$ Model 1: adjusted for age, country of residence, and physical activity at baseline

Model 2: additionally adjusted for educational level, smoking, alcohol consumption, weight status, number of chronic diseases, and use of analgesics and/or anti-inflammatory and anti-rheumatic products

${ }^{\mathrm{d}}$ The longitudinal associations are displayed per 10 units increase in pain severity
Table 4 Longitudinal associations between pain variability and physical activity, stratified by sex ${ }^{\mathrm{a}-\mathrm{c}}$

\begin{tabular}{lllllll}
\hline & Men & & & Women \\
\cline { 2 - 3 } \cline { 6 - 7 } & Ratio $(95 \% \mathrm{Cl})$ & $p$-value & & Ratio $(95 \% \mathrm{Cl})$ & $p$-value \\
\hline Model 1 & $1.15(0.98-1.33)$ & 0.08 & & $0.93(0.85-1.02)$ & 0.14 \\
Model 2 & $1.18(1.01-1.38)$ & 0.04 & & $0.94(0.86-1.03)$ & 0.21 \\
\hline
\end{tabular}

a Abbreviation: Cl Confidence Interval

${ }^{b}$ In these analyses, the individual joint pain standard deviation score (calculated from the three two-week pain calendars at baseline, 6 months

follow-up and 12-18 months follow-up) was the main determinant and

physical activity at 12-18 months follow-up the outcome measure

cModel 1: adjusted for age and country of residence

Model 2: additionally adjusted for educational level, smoking, alcohol

consumption, weight status, number of chronic diseases, and use of analgesics and/or anti-inflammatory and anti-rheumatic products

Furthermore, the observed sex-differences in the association between pain severity and PA could be explained by socio-cultural beliefs or gender role expectations regarding how men and women should respond to pain. It might be less accepted from men than from women when they adjust their PA levels because of greater pain severity $[38,43]$. The influencing role of different coping strategies in the association between pain and PA in older men and women with OA should be addressed in future research.

The results of the present study showed that greater pain variability was associated with more PA in older men. On forehand, we expected that greater pain variability would mean more unpredictability, which may lead to fear of sudden increases in pain, that would lead individuals to reduce their activities and activity levels [18]. A possible explanation for the current finding could be that older men with OA, in periods of less pain, become considerably more physically active, until pain increases again. As a result, more pain fluctuations could lead to more PA.

Although the results of the present study suggest that pain severity and pain variability affect PA levels of older adults with OA, it could also be that PA affects pain [7, 9]. It has been suggested that physical activity (e.g., supervised exercise therapy) could strengthen the muscles around an affected joint, which helps to stabilize the joint and to absorb most of the forces presented to the joint, resulting in less pain [44]. Future research is needed to consider the possibility of reversed causality, by examining how PA at baseline influences pain at follow-up and what the potential implications of this relationship are for PA at follow-up.

The EPOSA-project is a large-scale study with a pre-harmonized dataset including prospective cohort data from older adults with clinical knee, hand and/or hip OA from six European countries [27]. An important strength of our study is that the assessment of clinical OA was performed according to a uniform protocol in all six countries. Another strength is that the study 
sample was drawn from the general population. Consequently, our study includes data from individuals with mild and severe OA. In addition, our study includes data from persons seeking care and not seeking care. This increases generalizability of our results. Furthermore, a strength of this study is the assessment of day-to-day pain variability using several two-week pain calendars over a large time period. This is a novel and promising way of investigating joint pain in OA [26].

The current study has some limitations as well. Firstly, the participants in our study were not asked about other types of pain that they may have experienced at the same time as their joint pain. As a consequence, we were unable to control for this potentially important confounder. Secondly, there was no exact overlap between the timing of the pain assessments and the timing of the PA assessments. In the present study, the pain calendar at 12-18 months follow-up was completed in the two weeks after the PA assessment, whereas PA was our dependent variable. However, the pain calendar started the day after the 12-18 months follow-up interview, and consequently, the time period between these assessments was quite short. Excluding data from the third pain calendar would not only reduce the statistical power of our analyses, but would also mean that PA would be assessed six months after the last pain calendar (and 12-18 months for Spain), which is a much longer period. The sensitivity analyses in which the 12-18 months pain calendar was left out showed similar results as the main analyses. Thirdly, the $\mathrm{SD}$ of the individual pain scores from all available days of three two-week pain calendars was used as measure of within-person pain variability in this study. It has been suggested that these SD scores may suffer from relatively low reliability [45], and this might be more pronounced if the number of observations is low. In the current study, we combined three two-week pain calendars in order to make the reliability of the pain variability measure as high as possible. For the majority of the participants, the individual SD was calculated from 42 observations. Finally, PA was self-reported using the LAPAQ [29]. It has been suggested that self-reported PA has some limitations compared to objectively measured PA, such as susceptibility to overestimation, recall, and culture difference bias, especially in older adults $[46,47]$.

Overall, the current study may help to better understand pain patterns in older adults with $\mathrm{OA}$ and how these patterns affect daily functioning of these individuals in terms of PA. However, the observed sex differences in the various associations should be studied in more detail and need replication in future research. Ultimately, health care professionals could use the insights gained from such studies in clinical encounters to tailor recommendations for the timing of pain medication use and PA behavioral strategies.
To obtain more detailed knowledge about the association between OA-related pain and PA, future research could objectively assess PA using accelerometers and let participants fill in a pain calendar at the same time. Furthermore, these studies could focus on whether pain variability is associated with specific activities in men and women, and whether there are differences in other factors that may cause sex differences in the various associations.

\section{Conclusions}

In conclusion, the present study showed that greater pain severity was associated with less PA in older women. Furthermore, the findings of this study show that greater pain variability was associated with more PA in older men.

\section{Abbreviations}

AUSCAN: Australian/Canadian Hand Osteoarthritis Index (AUSCAN);

$\mathrm{Cl}$ : Confidence interval; EPOSA: European Project on OSteoArthritis; IBM SPSS: International Business Machines Corporation Statistical Package for the Social Sciences; IQR: Interquartile range; kcal: Kilocalories; kg/m²: Kilogram per square meter; LAPAQ: Longitudinal Aging Study Amsterdam Physical Activity Questionnaire; Ln: Natural logarithm; n: Number of participants; $\mathrm{N}_{\text {exp }}$ : Number of persons in a specific age/sex category in the European population; $\mathrm{N}_{\text {obs }}$ : Number of persons in a specific age/sex category in the cohort; OA: Osteoarthritis; PA: Physical activity; SD: Standard deviation; W: Weight; WOMAC: Western Ontario and McMaster Universities OA Index

\section{Acknowledgements}

We are very grateful to all participants of the EPOSA study for their valued contributions.

The EPOSA research group: Germany: T. Nikolaus ${ }^{\dagger}$ (Principal Investigator), R. Peter, M.D. Denkinger and F. Herbolsheimer; Italy: S. Maggi (Principal Investigator), S. Zambon, P. Siviero, F. Limongi and M. Noale; the Netherlands (coordinating center): D.J.H. Deeg (Principal Investigator), E.J. Timmermans, S. van der Pas, L.A. Schaap and N.M. van Schoor; Spain: Á. Otero (Principal Investigator), M.V. Castell, M. Sánchez-Martínez and R. Quieipo; Sweden: N.L. Pedersen (Principal Investigator) and R. Broumandi; United Kingdom: E.M. Dennison (Principal Investigator), C. Cooper, M.H. Edwards and C. Parsons.

\section{Funding}

The Indicators for Monitoring COPD and Asthma - Activity and Function in the Elderly in Ulm study (IMCA - ActiFE) was supported by the European Union [No.: 2005121] and the Ministry of Science, Baden-Württemberg. The Italian cohort study is part of the National Research Council Project on Aging (PNR). The Longitudinal Aging Study Amsterdam (LASA) is financially supported by the Dutch Ministry of Health, Welfare and Sports, Directorate of Long-term Care. The Penagrande study was partially supported by the National Fund for Health Research (Fondo de Investigaciones en Salud) of Spain [project numbers FIS PI 05/1898; FIS RETICEF RD06/0013/1013 and FIS PS09/02143]. The Swedish Twin Registry is supported in part by the Swedish Ministry of Higher Education. The Hertfordshire Cohort Study is funded by the Medical Research Council of Great Britain, Arthritis Research UK, the British Heart Foundation and the International Osteoporosis Foundation. The funding sources had no role in the study design, the collection, analysis and interpretation of the data, in the writing of the report and in the decision to submit the article for publication.

\section{Availability of data and materials}

Data from the European Project on OSteoArthritis (EPOSA; www.eposa.org) are available for use for specific research questions provided that an agreement is made up.

\section{Authors' contributions}

EJT drafted the article, with the help of EJdK, DJHD and LAS. NMvS, SvdP, MDD, EMD, SM, NLP, AO, RP, CC, PS, MVC, FH, ME and FL revised the article critically for important intellectual content. EJT, NMvS, SvdP, MDD, EMD, SM, 
NLP, AO, RP, CC, PS, MVC, FH, ME, FL, DJHD and LAS made substantial contributions to study conception and design and made substantial contributions to acquisition of data. EJT, EJdK, DJHD and LAS made substantial contributions to analyses and interpretation of data. All authors approved the final version of the article.

\section{Ethics approval and consent to participate}

All six cohort studies were approved by the Ethical Review Boards of the respective institutions (Germany: Ethical Committee of UIm University; Italy: Ethics Committee ULSS7; the Netherlands: Medical Ethical Committee of the VU University medical center; Spain: Ethical Committee for Clinical Research of University Hospital La Paz of Madrid; Sweden: Ethics Board of Karolinska Institute; United Kingdom: The Hertfordshire Research Ethics Committee). All participants gave written informed consent prior to the start of the study.

\section{Consent for publication}

Not applicable.

\section{Competing interests}

The authors declare that they have no conflicts of interest.

\section{Publisher's Note}

Springer Nature remains neutral with regard to jurisdictional claims in published maps and institutional affiliations.

\section{Author details}

${ }^{1}$ Department of Epidemiology and Biostatistics, Amsterdam University Medical Center, VU University Medical Center, Amsterdam Public Health Research Institute, De Boelelaan 1089A, 1081HV, Amsterdam, Netherlands. ${ }^{2}$ Geriatric Research Unit / Institute of Epidemiology and Medical Biometry, AGAPLESION Bethesda Hospital, UIm University, Ulm, Germany. ${ }^{3} \mathrm{MRC}$ Lifecourse Epidemiology Unit, University of Southampton, Southampton General Hospital, Southampton, UK. ${ }^{4}$ National Research Council, Aging Branch, Institute of Neuroscience, Padova, Italy. ${ }^{5}$ Department of Medical Epidemiology and Biostatistics, Karolinska Institute, Stockholm, Sweden. ${ }^{6}$ Department of Preventive Medicine and Public Health, Unit of Primary Care and Family Medicine, Faculty of Medicine, Universidad Autonoma de Madrid, Madrid, Spain. ${ }^{7}$ Institute of the History, Philosophy and Ethics of Medicine, Ulm University, Ulm, Germany. ${ }^{8}$ Department of Health Sciences, Faculty of Science, Amsterdam Public Health Research Institute, Vrije Universiteit Amsterdam, Amsterdam, Netherlands.

\section{Received: 23 August 2018 Accepted: 21 December 2018}

\section{Published online: 05 January 2019}

\section{References}

1. Woolf AD, Pfleger B. Burden of major musculoskeletal conditions. Bull World Health Organ. 2003;81:646-56.

2. Brooks PM. Impact of osteoarthritis on individuals and society: how much disability? Social consequences and health economic implications. Curr Opin Rheumatol. 2002;14:573-7.

3. Vos T, Flaxman AD, Naghavi M, Lozano R, Michaud C, Ezzati M, et al. Years lived with disability (YLDs) for 1160 sequelae of 289 diseases and injuries 1990-2010: a systematic analysis for the global burden of disease study 2010. Lancet. 2012;380:2163-96

4. Parry E, Ogollah R, Peat G. Significant pain variability in persons with, or at high risk of, knee osteoarthritis: preliminary investigation based on secondary analysis of cohort data. BMC Musculoskelet Disord. 2017:18:80.

5. Rolland JS. Chronic illness and the life cycle: a conceptual framework. Fam Process. 1987;26:203-21.

6. Dunlop DD, Semanik P, Song J, Manheim LM, Shih V, Chang RW. Risk factors for functional decline in older adults with arthritis. Arthritis Rheum. 2005;52: $1274-82$.

7. Dunlop DD, Song J, Semanik PA, Sharma L, Chang RW. Physical activity and functional performance in the osteoarthritis initiative: a graded relationship. Arthritis Rheum. 2011;61:127-36.

8. Nelson AE, Allen KD, Golightly YM, Goode AP, Jordan JM. A systematic review of recommendations and guidelines for the management of osteoarthritis: the chronic osteoarthritis management initiative of the U.S. bone and joint initiative. Semin Arthritis Rheum. 2014;43:701-12.
9. Vignon E, Valat J, Rossignol M, Avouac B, Rozenberg S, Thoumie P, et al. Osteoarthritis of the knee and hip and activity: a systematic international review and synthesis (OASIS). Joint Bone Spine. 2006;73:442-55.

10. White DK, Lee J, Song J, Chang RW, Dunlop DD. Potential functional benefit from light intensity physical activity in knee osteoarthritis. Am J Prev Med. 2017:53:689-96.

11. De Groot IB, Bussmann JB, Stam HJ, Verhaar JA. Actual everyday physical activity in patients with end-stage hip or knee osteoarthritis compared with healthy controls. Osteoarthr Cartil. 2008;16:436-42.

12. Farr JN, Going SB, Lohman TG, Rankin L, Kasle S, Cornett M, et al. Physical activity levels in early knee osteoarthritis patients measured by accelerometry. Arthritis Rheum. 2008;59:1229-36.

13. Herbolsheimer F, Schaap LA, Edwards MH, Maggi S, Otero Á, Timmermans EJ, et al. Physical activity patterns among older adults with and without knee osteoarthritis in six European countries. Arthritis Care Res (Hoboken). 2016;68:228-36

14. Stubbs B, Binnekade TT, Soundy A, Schofield P, Huijnen IP, Eggermont LH Are older adults with chronic musculoskeletal pain less active than older adults without pain? A systematic review and meta-analysis. Pain Med. 2013;14:1316-31.

15. Veenhof $C$, Huisman PA, Barten JA, Takken T, Pisters MF. Factors associated with physical activity in patients with osteoarthritis of the hip or knee: a systematic review. Osteoarthr Cartil. 2012;20:6-12.

16. Wallis JA, Webster KE, Levinger P, Taylor NF. What proportion of people with hip and knee osteoarthritis meet physical activity guidelines? A systematic review and meta-analysis. Osteoarthr Cartil. 2013;21:1648-59.

17. Holden MA, Nicholls EE, Young J, Hay EM, Foster NE. Role of exercise for knee pain: what do older adults in the community think? Arthritis Care Res (Hoboken). 2012;64:1554-64.

18. Pettursdottir U, Arnadottir SA, Halldorsdottir S. Facilitators and barriers to exercising among people with osteoarthritis: a phenomenological study. Phys Ther. 2010;90:1014-25.

19. Stubbs B, Hurley M, Smith T. What are the factors that influence physical activity participation in adults with knee and hip osteorthritis? A systematic review of physical activity correlates. Clin Rehabil. 2015;29:80-94.

20. Allenyzx KD, Coffmanyk CJ, Golightlyy YM, Stechuchaky KM, Keefe FJ. Daily pain variations among patients with hand, hip, and knee osteoarthritis. Osteoarthr Cartil. 2009:17:1275-82.

21. Collins JE, Katz JN, Dervan EE, Losina E. Trajectories and risk profiles of pain in persons with radiographic symptomatic knee osteoarthritis: data from the osteoarthritis initiative. Osteoarthr Cartil. 2014;22:622-30.

22. Murphy SL, Kratz AL, Williams DA, Geisser ME. The association between symptoms between symptoms, pain coping strategies, and physical activity among people with symptomatic knee and hip osteoarthritis. Front Psychol. 2012:3:326.

23. Murphy SL, Smith DM, Clauw DJ, Alexander NB. The impact of momentary pain and fatigue on physical activity in women with osteoarthritis. Arthritis Rheum. 2008:59:849-56.

24. Bellamy N. WOMAC osteoarthritis index: a user's guide. Ontario, Canada: London Health Services; 1996.

25. Bellamy N, Campbell J, Haraoui B, Gerecz-Simon E, Buchbinder R, Hobby K, et al. Clinimetric properties of the AUSCAN osteoarthritis hand index: an evaluation of reliability, validity, and responsiveness. Osteoarthr Cartil. 2002; 20:863-9.

26. De Koning EJ, Timmermans EJ, Van Schoor NM, Stubbs B, Van den Kommer TN, Dennison EM, et al. Within-person pain variability and mental health in older adults with osteoarthritis: an analysis across six European cohorts. J Pain. 2018;19:690-8

27. Van der Pas S, Castell MV, Cooper C, Denkinger M, Dennison EM, Edwards $\mathrm{MH}$, et al. European project on osteoarthritis: design of a six-cohort study on the personal and societal burden of osteoarthritis in an older European population. BMC Musculoskelet Disord. 2013;14:138.

28. Altman RD. Classification of disease: osteoarthritis. Seminars Arthritis Rheum. 1991;20:40-7.

29. Stel VS, Pluijm SMF, Deeg DJH, Smit JH, Bouter LM, Lips P. Functional limitations and poor physical performance as independent risk factors for self-reported fractures in older persons. Osteoporos Int. 2004;15:742-50.

30. Molton IR, Terrill AL. Overview of persistent pain in older adults. Am Psychol. 2014;69:197-207.

31. Ho A, Ashe MC, Delongis A Graf P, Khan KM, Hoppmann CA. Gender differences in pain-physical activity linkages among older adults: lessions 
learned from daily life approaches. Pain Res Manag. 2016;2016. https://doi. org/10.1155/2016/1931590.

32. Peacock S, Patel S. Cultural influences on pain. Reviews in Pain. 2008;1:6-9.

33. World Health Organization. Obesity: preventing and managing the global epidemic. Report of a WHO Consultation (WHO Technical Report Series 894). Geneva, Switzerland: World Health Organization; 2000.

34. Eurostat. Database, population, demography, national data, population. [Internet website, accessed for the EPOSA-project: February, 2013]. ec. europa.eu/eurostat/web/population-demography-migration-projections/ population-data/database.

35. Aiken LS, West SG. Multiple regression: testing and interpreting interactions. Thousand Oaks, United States of America: SAGE Publications, Inc; 1991.

36. Angst F, Aeschlimann A, Stucki G. Smallest detectable and minimal clinically important differences of rehabilitation intervention with their implications for required sample sizes using WOMAC and SF-36 quality of life measurement instruments in patients with osteoarthritis of the lower extremities. Arthritis Rheum. 2001:45:384-91.

37. Corp IBM. IBM SPSS statistics for windows, version 22.0. IBM Corp: Armonk, New York, United States of America; 2013.

38. Bartley EJ, Fillingim RB. Sex differences in pain: a brief review of clinical and experimental findings. Br J Anaesth. 2013;111:52-8.

39. Sullivan MJ, Thorn B, Haythornthwaite JA, Keefe F, Martin M, Bradley LA, et al. Theoretical perspectives on the relation between catastrophizing and pain. Clin J Pain. 2001;17:52-64

40. Bandura A. Self-efficacy: toward a unifying theory of behavioral change. Psychol Rev. 1977:84:191-215.

41. Keefe FJ, Brown GK, Wallston KA, Caldwell DS. Coping with rheumatoid arthritis pain: catastrophizing as a maladaptive strategy. Pain. 1989;37:51-6.

42. Jackson T, lezzi T, Gunderson J, Nagasaka T, Fritch A. Gender differences in pain perception: the mediating role of self-efficacy beliefs. Sex Roles. 2002; 47:561-8.

43. Wise EA, Price DD, Myers CD, Heft MW, Robinson ME. Gender role expectations of pain: relationship to experimental pain perception. Pain. 2002;96:335-42.

44. Bosomworth NJ. Exercise and knee osteoarthritis: benefit or hazard? Can Fam Physician. 2009;55:871-8.

45. Wang LP, Grimm KJ. Investigating reliabilities of intraindividual variability indicators. Multivar Behav Res. 2012;47:771-802.

46. Sallis JF, Saelens BE. Assessment of physical activity by self-report: status, limitations, and future directions. Res Q Exerc Sport. 2000;71:S1-S14.

47. Viken H, Petter Aspvik NH, Zisko N, Wisløff U, Stensvold D. Correlates of objectively measured physical activity among Norwegian older adults: the generation 100 study. J Aging Phys Act. 2016;24:369-75.

Ready to submit your research? Choose BMC and benefit from:

- fast, convenient online submission

- thorough peer review by experienced researchers in your field

- rapid publication on acceptance

- support for research data, including large and complex data types

- gold Open Access which fosters wider collaboration and increased citations

- maximum visibility for your research: over $100 \mathrm{M}$ website views per year

At $\mathrm{BMC}$, research is always in progress.

Learn more biomedcentral.com/submissions 